\title{
Radiation From an Infinite Axial Slot on a Cir- cular Cylinder Clad With Magnetoplasma
}

\author{
Phillippe de Marchin and G. Tyras
}

Department of Electrical Engineering, The University of Arizona, Tucson, Ariz.

(Received October 13, 1964; revised November 23, 1964)

\begin{abstract}
This paper is concerned with the investigation of the effects of a magnetoplasma layer on the radiation properties of an infinite axial slot on a circular cylinder when a finite magnetostatic field is in the axial direction. Formulas have been obtained for the fields everywhere around the cylinder and for any thickness of the magnetoplasma. The solution is simplified by a first order approximation, valid only for small thicknesses of the layer. For a small " $k_{0} a$ " the harmonic series converges well and is used for numerical computations. For large " $k_{0} a$ " the convergence is improved by applying the Watson's transformation. Distortion, attenuation, and asymmetry of the radiation pattern are shown as a function of the strength of the magnetostatic field. It is found that the reversal of the orientation of the magnetostatic field produces a shift of the pattern to a mirror-image position.
\end{abstract}

\section{Introduction}

There has been a considerable interest in the recent years in the problem of radiation from apertures covered with a plasma sheath. Such a sheath is formed around the body of a hypersonic vehicle during its re-entry into the earth's atmosphere and its electromagnetic effects may cause sufficient distortion and/or attenuation of the antenna pattern to make the communication with the ground station unreliable.

The direct approach of alleviating the problem simply amounts to increasing the operating frequency beyond the highest plasma frequency within the plasma sheath. This, however, may sometimes not be practical because it could possibly lead to operating frequencies higher than those of the present systems. Other ways of alleviating the problem have been proposed, one of which is the application of a strong magnetostatic field in the neighborhood of the radiating aperture [Harley and Tyras, 1961; Hodora, 1961 and 1963; Shore and Meltz, 1962]. Practical aspects of producing a sufficiently strong magnetic field on a hypersonic vehicle aside, such a scheme can indeed reduce the propagation losses through the sheath.

An outstanding contribution to the theory of radiation from arbitrary apertures on perfectly conducting cylinders was made by Silver and Saunders [1950]. Special shapes of apertures such as half-wave longitudinal and axial slots have been treated extensively and radiation patterns are available. Slots on circular cylinders with isotropic dielectric coating have been studied by Wait [1959]. More recently, the solution to the problem of radiation from an arbitrary slot on a plasma coated cylinder has been found by Yeh [1964], while Wait [1963] considered the transverse propagation in a cylindrically stratified plasma.
The present paper deals with the problem of radiation from an infinite axial slot on a circular cylinder clad with a uniform and homogeneous magnetoplasma layer when the magnetostatic field is parallel to the axis of the cylinder. This particular configuration makes it possible to arrive at a solution that can be written explicitly in terms of the pertinent magnetoplasma parameters and without recourse to their limiting values [Shore and Meltz, 1962; Hodora, 1963]. This is the consequence of the fact that the resulting differential equations are still of second order.

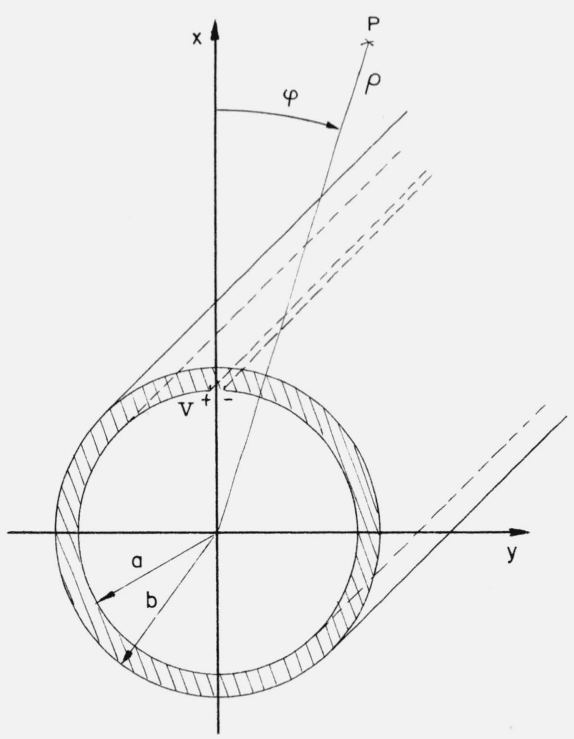

FigURE 1. Geometrical configuration of the problem. 


\section{Formulation of Solution}

The geometrical configuration of the problem is shown in figure 1 . The plasma coating extends from radius ' $a$ ' to ' $b$ '. It is assumed to be homogeneous, lossless, and in the case of the magnetostatic field, $H_{\mathrm{DC}}$, oriented in the positive $z$-direction, representable by the permittivity tensor

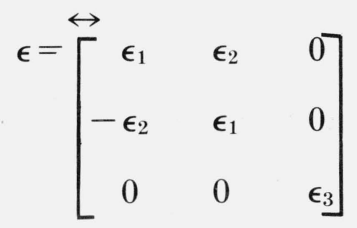

where

$$
\begin{gathered}
\epsilon_{1}=1-\frac{\omega_{P}^{2}}{\omega^{2}-\omega_{H}^{2}} \quad \epsilon_{2}= \pm 1 \frac{\left|\omega_{H}\right| \omega_{P}^{2}}{\omega\left(\omega^{2}-\omega_{H}^{2}\right)} \\
\epsilon_{3}=1-\frac{\omega_{P}^{2}}{\omega^{2}} \quad \omega_{H}=\frac{e \mu_{0} H_{\mathrm{DC}}}{m} \quad \omega_{P}^{2}=\frac{e^{2} N}{m \epsilon_{0}} .
\end{gathered}
$$

The problem can be solved using only the axial component of the magnetic field. The resulting wave equation to be satisfied in the magnetoplasma is

$$
\left(\nabla x \stackrel{\leftrightarrow}{-1} \nabla x-k_{0}^{2}\right) H_{z}=0 .
$$

Following Wait [1961], we shall seek a solution for $H_{z}$ in the form

$$
H_{z}=\sum_{-\infty}^{+\infty} e^{i m \phi} h_{m z},
$$

and it will follow that $h_{m z}$ must satisfy the Bessel differential equation

$$
\left(\frac{\partial^{2}}{\partial \rho^{2}}+\frac{1}{\rho} \frac{\partial}{\partial \rho}-\frac{m^{2}}{\rho^{2}}+k_{0}^{2} \frac{\epsilon_{T}^{2}}{\epsilon_{1}}\right) h_{m z}=0,
$$

where $\epsilon_{T}^{2}=\epsilon_{1}^{2}+\epsilon_{2}^{2}$.

Once the axial component of the magnetic field is determined, the components of the electric field can be found from the relation

$$
\vec{E}=\frac{i}{\omega \epsilon_{0}} \stackrel{\leftrightarrow}{\epsilon^{-1}} \nabla x \vec{H}
$$

The boundary conditions require that $h_{m z}$ and $e_{m \phi}$ be continuous at the plasma-air interface, i.e.,

$$
Q_{m} J_{m}(k b)+E_{m} N_{m}(k b)=F_{m} H_{m}\left(k_{0} b\right)
$$

$$
\begin{array}{r}
Q_{m}\left[-\frac{i m \epsilon_{2}}{b \epsilon_{T}^{2}} J_{m}(k b)+\right. \\
\left.\frac{\epsilon_{1} k}{\epsilon_{T}^{2}} J_{m}^{\prime}(k b)\right]+E_{m}\left[-\frac{i m \epsilon_{2}}{b \epsilon_{T}^{2}} N_{m}(k b)\right. \\
\left.+\frac{\epsilon_{1} k}{\epsilon_{T}^{2}} N_{m}^{\prime}(k b)\right]=F_{m} k_{0} H_{m}^{\prime}\left(k_{0} b\right)
\end{array}
$$

where $k_{0}=\omega \sqrt{\epsilon_{0} \mu_{0}}$ and $k=k_{0} \epsilon_{T} / \sqrt{\epsilon_{1}}$.

Moreover, on the surface of the cylinder $E_{\phi}$ must be equal to the expression of the source, $E_{\phi}=\frac{V \delta(\phi)}{a}$, which implies

$$
\begin{aligned}
Q_{m}[ & \left.-\frac{i m \epsilon_{2}}{a \epsilon_{T}^{2}} J_{m}(k a)+\frac{\epsilon_{1} k}{\epsilon_{T}^{2}} J_{m}^{\prime}(k a)\right] \\
& +E_{m}\left[-\frac{i m \epsilon_{2}}{a \epsilon_{T}^{2}} N_{m}(k a)+\frac{\epsilon_{1} k}{\epsilon_{T}^{2}} N_{m}^{\prime}(k a)\right]=\frac{i \omega \epsilon_{0} V}{2 \pi a} .
\end{aligned}
$$

Solving (7), (8), and (9) for $Q_{m}, E_{m}$, and $F_{m}$, we obtain the solution for the fields in free space:

$$
\begin{aligned}
H_{z} & =\sum_{-\infty}^{+\infty} e^{i m \phi} \boldsymbol{F}_{m} H_{m}^{(1)}\left(k_{0} \rho\right) \\
E_{\phi} & =\frac{1}{i \omega \epsilon_{0}} \frac{\partial H_{z}}{\partial \rho} \\
E_{\rho} & =-\frac{1}{i \omega \epsilon_{0}}-\frac{1 \partial H_{z}}{\partial \phi}
\end{aligned}
$$

with

$$
F_{m}=-\frac{i \omega \epsilon_{0} V \epsilon_{1}}{\pi^{2} a b \epsilon_{T}^{2}} \frac{1}{D_{m}}
$$

and

$$
\begin{array}{r|c|c}
D_{m}= & J_{m}(k b) & H_{m}(k b) \\
\hline-\frac{i m \epsilon_{2}}{b \epsilon_{T}^{2}} J_{m}(k b) & -\frac{i m \epsilon_{2}}{b \epsilon_{T}^{2}} N_{m}(k b) & \\
+\frac{\epsilon_{1} k}{\epsilon_{T}^{2}} J_{m}^{\prime}(k b) & +\frac{\epsilon_{1} k}{\epsilon_{T}^{2}} N_{m}^{\prime}(k b) & k_{0} H_{m}^{\prime}\left(k_{0} b\right) \\
\hline-\frac{i m \epsilon_{2}}{\epsilon_{T}^{2}} J_{m}(k a) & -\frac{i m \epsilon_{2}}{a \epsilon_{T}^{2}} N_{m}(k a) & \\
+\frac{\epsilon_{1} k}{\epsilon_{T}^{2}} J_{m}^{\prime}(k a) & +\frac{\epsilon_{1} k}{\epsilon_{T}^{2}} N_{m}^{\prime}(k a) & 0
\end{array}
$$

This result can be partially checked in many ways. If the thickness of the coating goes to zero or the components of the permittivity tensor take the values $\epsilon_{1}=\epsilon_{3}=1, \epsilon_{2}=0$, then

$$
F_{m}=\frac{i V}{2 \pi a \eta_{0} H_{m}^{(1)^{\prime}}\left(k_{0} a\right)}
$$

which is the well-known solution for the slot without coating. Now if $\epsilon_{1}=\epsilon_{3}=\epsilon_{r}, \epsilon_{2}=0$ then $F_{m}$ is found to be 


$$
F_{m}=\frac{-i V}{\pi^{2} \eta_{0} k a b} \cdot\left|\begin{array}{lll}
J_{m}(k b) & N_{m}(k b) & H_{m}^{(1)}\left(k_{0} b\right) \\
\eta_{r} J_{m}^{\prime}(k b) & \eta_{r} N_{m}^{\prime}(k b) & H_{m}^{(1)}\left(k_{0} b\right) \\
J_{m}^{\prime}(k a) & N_{m}^{\prime}(k a) & 0
\end{array}\right|
$$

which is the solution given by Wait [1959] for the radiation in the equatorial plane of a finite axial slot on a cylinder clad with isotropic dielectric.

\section{Assmmetry and Mirror Image Property}

Equations (10), (13), and (14) can be put in the following form:

$$
\begin{aligned}
& H_{z}=C \sum_{m=0}^{\infty} \\
& \frac{\left[D_{-m}+(-1)^{m} D_{m}\right] \cos m \phi+i\left[D_{-m}-(-1)^{m} D_{m}\right] \sin m \phi}{D_{m} D_{-m}} H_{m}^{(1)}\left(k_{0} \rho\right)
\end{aligned}
$$

and since by virtue of (14) $D_{-m} \neq(-1)^{m} D_{m}$, the radiation pattern is not symmetric with respect to the $\phi=0$ plane.

If the direction of the steady magnetic field is reversed, $\epsilon_{2}$ changes its sign. Since the following relations hold

$$
\begin{aligned}
& D_{m,-\epsilon_{2}}=(-1)^{m} D_{-m, \epsilon_{2}} \\
& D_{-m,-\epsilon_{2}}=(-1)^{m} D_{m, \epsilon_{2}}
\end{aligned}
$$

it is seen that the same value for the magnetic field is obtained at an angle $-\phi$, and hence the radiation pattern shifts to a mirror image position.

This unusual phenomenon of asymmetry and mirror image property has been known for some time to exist in the related configurations involving magnetized ferrites. It was first observed experimentally by Angelakos and Korman [1956] in the case of a ferrite loaded waveguide and later confirmed analytically by Tyras and Held [1958].

\section{First Order Approximation}

Although the general solution obtained is valid for any magnetoplasma of arbitrary thickness, the usefulness of this result is, however, limited by its complexity. A great simplification of the result follows when the thickness of the magnetoplasma layer is allowed to be small. In this case the following approximations are valid, where $B_{m}$ represents any Bessel function,

$$
\begin{aligned}
& B_{m}(k a)=B_{m}(k b)-k t B_{m}^{\prime}(k b) \\
& B_{m}^{\prime}(k a)=B_{m}^{\prime}(k b)-k t B_{m}^{\prime \prime}(k b)
\end{aligned}
$$

provided that the thickness $t$ be small compared to $\frac{\lambda}{2 \pi}$ where $\lambda$ is the wavelengtt $i$ in plasma.

Neglecting all second order quantities which are small and using Wronskian and certain recurrence relations between Bessel functions, the following result is obtained

$$
H_{z}=\frac{i V}{2 \pi \eta_{0} b} \sum_{m=-\infty}^{+\infty} \frac{e^{i m \phi} H_{m}^{(1)}\left(k_{0} \rho\right)}{H_{m}^{(1)}\left(k_{0} b\right)+G_{m} H_{m}^{(1)}\left(k_{0} b\right)}
$$

with

$$
G_{m}=\frac{t}{b}\left[k_{0} a\left(1-\frac{m^{2}}{k^{2} b^{2}}+\frac{m^{2} \epsilon_{2}}{k_{0} b \epsilon_{T}^{2}}\right)+\frac{i m \epsilon_{2}}{\epsilon_{1}} \frac{H_{m}^{(1)}{ }^{\prime}\left(k_{0} b\right)}{H_{m}^{(1)}\left(k_{0} b\right)}\right] .
$$

Equation (20) is the expression of the field produced by an infinite slot on a cylinder having a surface impedance $Z_{m}=i \eta_{0} G_{m}$. If the plasma is identified with an isotropic dielectric and if $k b$ is large, then

$$
G_{m} \cong \frac{t}{b}\left(k_{0} a\right)\left(1-\frac{m^{2}}{k^{2} b^{2}}\right)
$$

which is the expression found by Wait [1959].

\section{Computation of Radiation Patterns}

The first order approximation solution has been programmed for a digital computer. Radiation patterns have been obtained, figures $2 \mathrm{a}-\mathrm{e}$, for different values of the magnetostatic field and for the following conditions

$$
\begin{aligned}
N & =8.5 \times 10^{10} \text { electrons } / \mathrm{cm}^{3} \\
\frac{t}{a} & =0.02 \\
k_{0} a & =3 \\
f & =150 \mathrm{Mc} / \mathrm{s} .
\end{aligned}
$$

There are several points worth mentioning:

(1) The asymmetry of the pattern is obvious for all values of the steady magnetic field. Such asymmetries have already been encountered in other problems involving plasma configurations.

(2) The shifting of the main lobe is considerable and can reach $90^{\circ}$ for certain values of the steady magnetic field. Many sidelobes are created in the shadow region of the antenna, due to a complex interaction of creeping waves.

(3) The impedance of the antenna varies widely. This can be estimated roughly from the magnitude of the radiation patterns.

(4) For very low and very high values of the magnetic field (under $100 \mathrm{G}$ and over 4,000 G) the radiation patterns are not affected much by the presence of plasma coating. When the value of the magnetostatic field is around $1430 \mathrm{G}$ the radiation pattern becomes very large and the antenna behaves like a line source, the impedance of which is very small. 


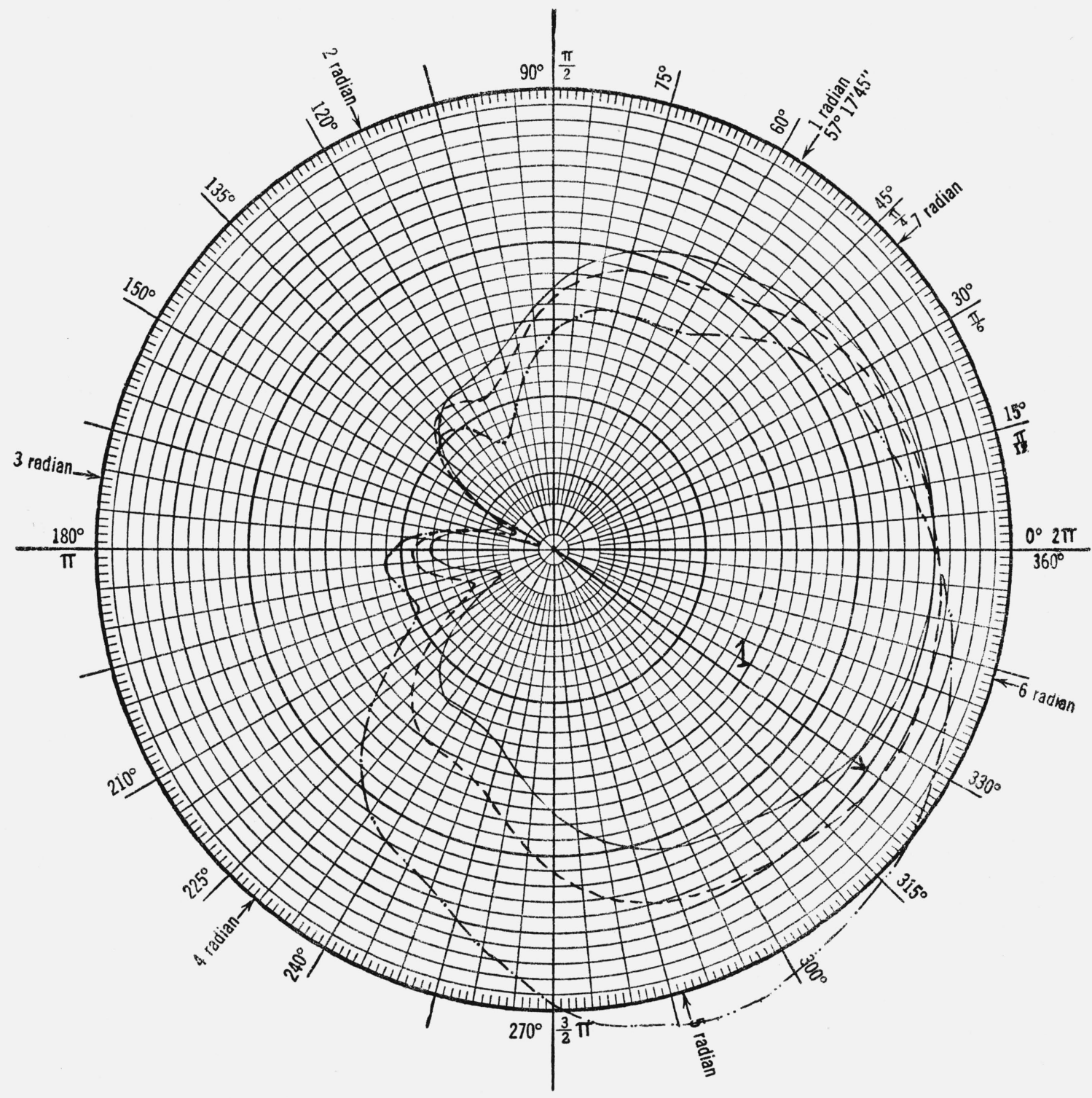

Isotropic Case

--100 Gauss

-..-300 Gauss

FIGURE 2a. Radiation patterns with the strength of the magnetostatic field as a variable parameter. 


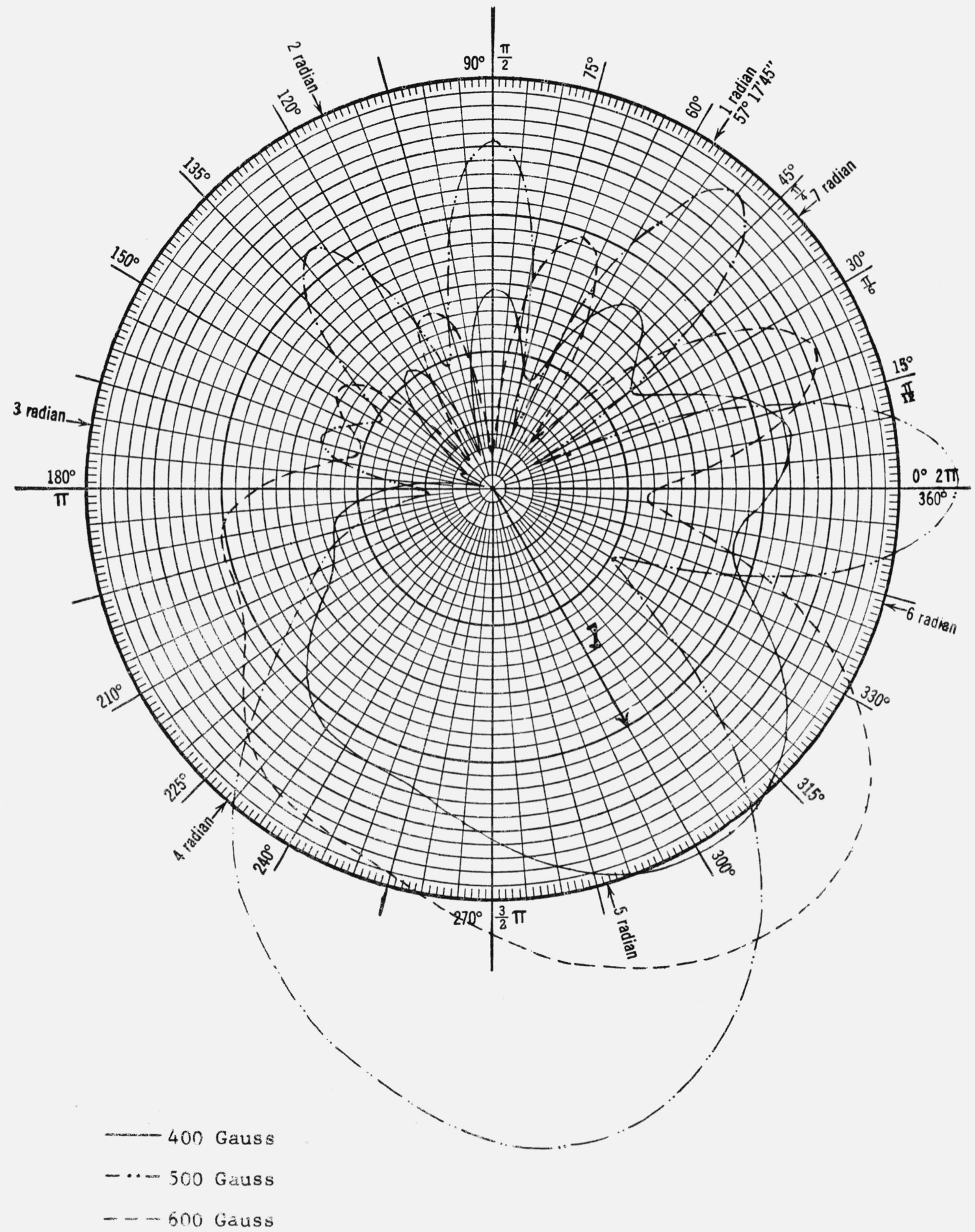

FIGURE 2b. Radiation patterns with the strength of the magnetostatic field as a variable parameter. 


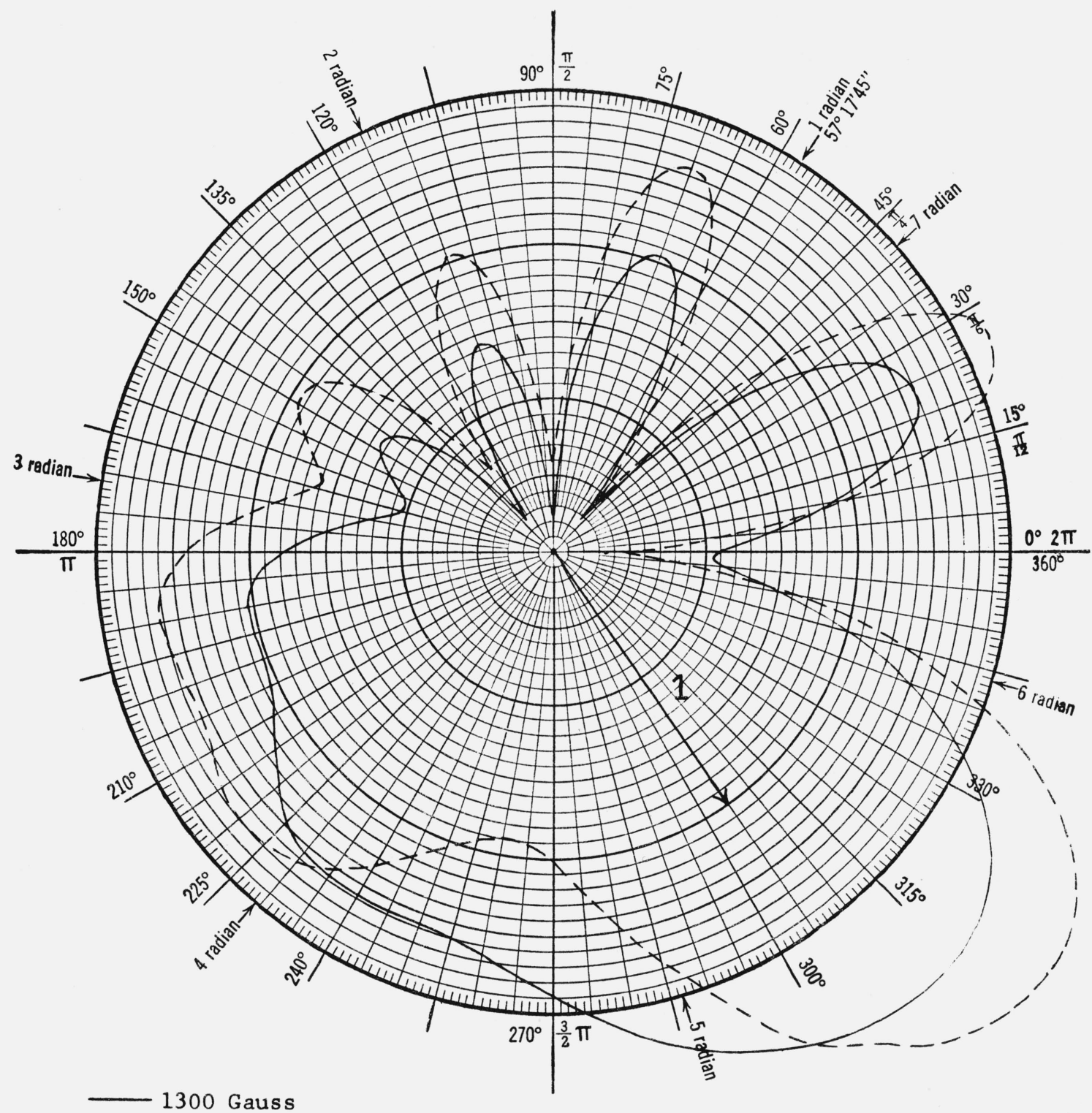

- - 1370 Gauss

FIGURE 2c. Radiation patterns with the strength of the magnetostatic field as a variable parameter. 


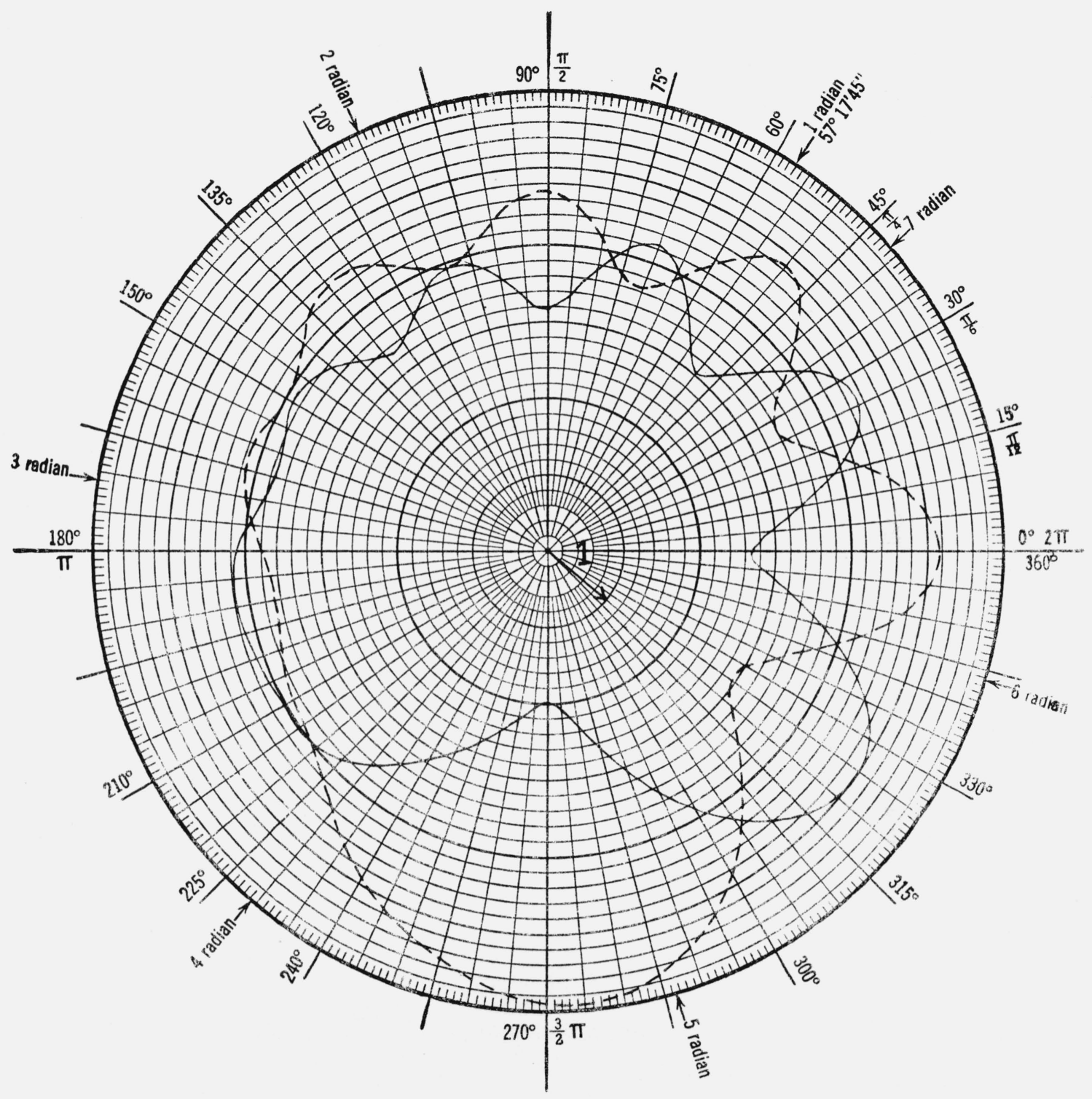

1420 Gauss

-... 1440 Gauss

FIGURE 2d. Radiation patterns with the strength of the magnetostatic field as a variable parameter.

\section{Application of Watson Transformation}

For large values of " $k_{0} a$ " the series (20) converges slowly. An alternate representation of the solution, the so-called "residue-series", can be obtained using the Watson transformation. It will follow that the residue series will be useful for numerical calculations when " $k_{0} a$ " is large. The solution $H_{z}$ can be written 


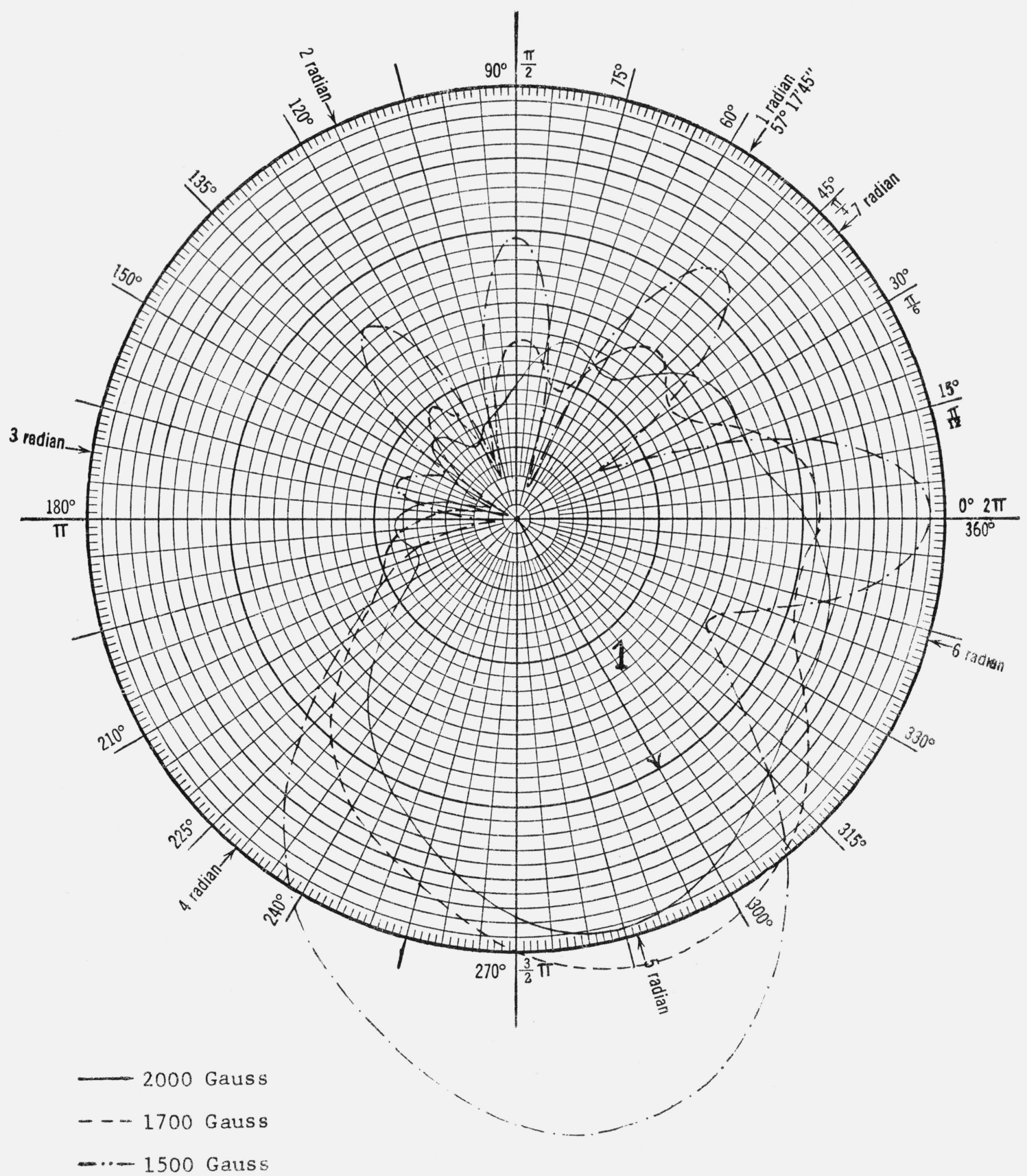

FIGURE 2e. Radiation patterns with the strength of the magnetostatic field as a variable parameter.

as follows

$H_{z}=-\frac{V}{4 \pi \eta_{0} a} \int_{\Gamma} \frac{H_{\nu}^{(1)}\left(k_{0} \rho\right)}{H_{\nu}^{(1)^{\prime}}\left(k_{0} a\right)+G_{\nu} H_{\nu}^{(1)}\left(k_{0} a\right.} \frac{e^{i \nu(\phi-\pi)} \sin \nu \pi}{\sin } d \nu$

where $\Gamma$ is the contour in the complex $\nu$-plane as shown in figure 3 . The contour $\Gamma$ can be supplemented by two semicircles which contribute nothing to the value of the integral in (23). The new contour encloses the poles of

$$
H_{\nu}^{(1)^{\prime}}\left(k_{0} a\right)+G_{\nu} H_{\nu}^{(1)}\left(k_{0} a\right)=0 .
$$

Referring to the case where the thickness of the layer is zero, we shall call the unperturbed poles the roots 
in the $\nu$-plane of

$$
H_{\nu}^{(1)^{\prime}}\left(k_{0} a\right)=0 .
$$

Since $G_{\nu}$ is small, we expect the "perturbed poles," solution of (24), to be located in the vicinity of the unperturbed poles, i.e., in the first and third quadrant. Use is made of the third order Debye approximation for the Hankel function of large argument

$$
H_{\nu}^{(1)}(x)=\frac{2 e^{-i \pi / 3}}{\pi}\left(\frac{6}{x}\right)^{1 / 3} A(z)
$$

where $A(z)$ is the Airy function defined as

$$
A(z)=\int_{0}^{\infty} \cos \left(t^{3}-z t\right) d t
$$

with

$$
z=(\nu-x)\left(\frac{6}{x}\right)^{1 / 3} e^{-i \pi / 3}
$$

Since $\mathrm{x} \cong \nu$, the approximation $\frac{\partial H_{\nu}}{\partial x}=-\frac{\partial H_{\nu}}{\partial x}$ is valid and it follows that

$$
\frac{H_{\nu}^{(1)^{\prime}}(x)}{H_{\nu}^{(1)}(x)}=-\frac{e^{-i \pi / 3}\left(\frac{6}{x}\right)^{1 / 3} A^{\prime}(z)}{A(z)}=-G_{\nu}
$$

with $x=k_{0} a$.

The left hand side of (29) is expected to be small since the thickness is small and therefore it can be expanded in a Taylor series about any zero $z_{0}$ of $A^{\prime}\left(z_{0}\right)=0$. Noting that the differential equation satisfied by $A(z)$ as defined in (27) is

$$
A^{\prime \prime}(z)+\frac{z}{3} A(z)=0,
$$

it can be shown that

$$
\frac{A^{\prime}(z)}{A(z)}=-\frac{z_{0}}{3}\left(z-z_{0}\right)-\frac{1}{6}\left(z-z_{0}\right)^{2}+\ldots .
$$

From (28) it follows that the perturbed $\nu$ pole is related to the unperturbed pole $\nu_{0}$ via the relation

$$
\nu=\nu_{0}+\left(\frac{6}{x}\right)^{-1 / 3} e^{i \pi / 3}\left(z-z_{0}\right)
$$

The next step is to combine relations (29), (31), and (32) and neglect second order quantities such as $\frac{t}{a}\left(z-z_{0}\right)$ or $\left(z-z_{0}\right)^{2} \ldots$ The following result is obtained.

$$
\frac{z_{0}}{3}\left(z-z_{0}\right)=\left(\frac{6}{k_{0} a}\right)^{-1 / 3} e^{i \pi / 3} \frac{t}{a} \frac{1}{k_{0} a}\left[\nu_{0}^{2} \frac{1-\epsilon_{1}}{\epsilon_{1}}+\nu_{0} \frac{i \epsilon_{2}}{\epsilon_{T}^{2}}\right]
$$

the value of the new pole being given by (32). Values of $z_{0}$ and corresponding $\nu_{0}$ can be found by Franz [1954].
The perturbed poles located in the third quadrant are found using relation (33), in which $\nu_{0}$ is replaced by $-\nu_{0}$, and with equation (32) replaced by

$$
\nu=-\nu_{0}-\left(\frac{6}{x}\right)^{-1 / 3} e^{i \pi / 3}\left(z-z_{0}\right) .
$$

The location of the new roots has been found by the method described above with the help of a digital computer for the following conditions: $\frac{t}{a}$ $=0.001, k_{0} a=200, f=10 \mathrm{Gc}$, the strength of the magnetostatic field being the variable parameter (fig. 4).

However, it is to be noted that, for $H_{\mathrm{DC}}=3450 \mathrm{G}, \epsilon_{1}$ is equal to zero, and hence the first order approximation is not valid and values of the magnetostatic field in that region must be discarded.

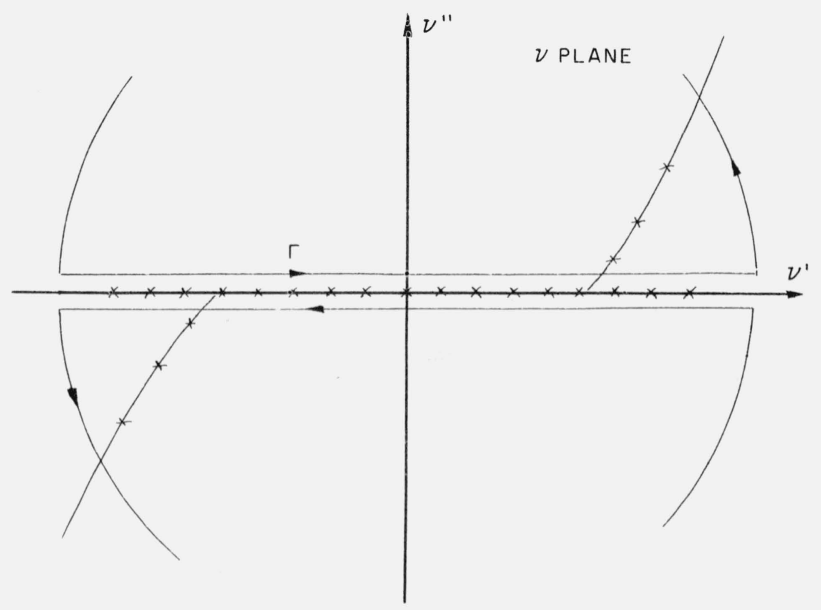

Figure 3. The $\Gamma$ contour in the complex $\nu$-plane.

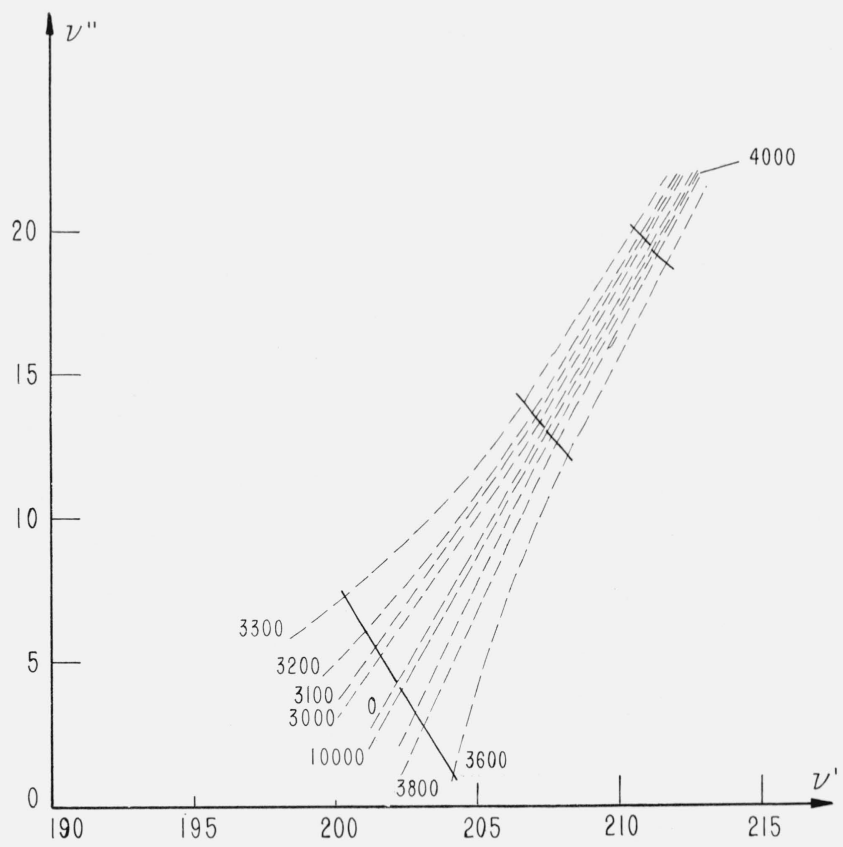

FIGURE 4. Location of the new poles in the complex $\nu$-plane as a function of the strength of the magnetostatic field in gauss. 


\section{Pattern Formulation in Shadow Region}

The final solution has been transformed by Watson transformation into the fast converging series

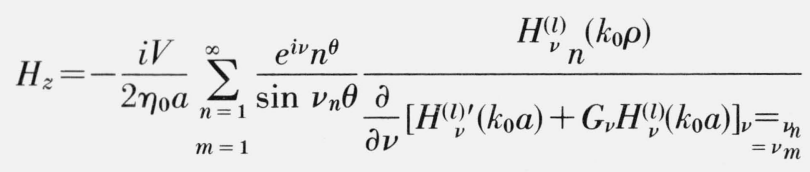

with $\theta=\phi-\pi$, and where the summation is made on the roots $\nu_{n}$ and $\nu_{m}$ of the first and third quadrant, respectively.

If we assume that $\nu_{n} \cong-\nu_{m}$ and moreover that the series converges fast enough so that only the first term has to be considered, then the pattern goes as

$$
\cos \nu_{n} \theta=\cos \nu^{\prime} \theta \cos h \nu^{\prime \prime} \theta+i \sin \nu^{\prime} \theta-\sin h \nu^{\prime \prime} \theta \cdot
$$

For the conditions given above, $\nu^{\prime} \cong 200, \nu^{\prime \prime} \cong 5$. In the region $\frac{\pi}{6}<|\theta|<\frac{\pi}{2}$ it follows that

$$
\cos h \nu^{\prime \prime} \theta=\sin h \nu^{\prime \prime} \theta
$$

and therefore the pattern goes as $e^{\nu^{\prime \prime \theta}}$. For small $\theta$, $\sin h \nu^{\prime \prime} \theta=0, \cos h \nu^{\prime \prime} \theta=1$, and the pattern goes as $\cos \nu^{\prime} \theta$, creating sidelobes having a width of approximately $\frac{\pi}{\nu^{\prime}}$ radians.

\section{References}

Angelakos, D. J., and M. M. Korman (Oct. 1956), Radiation from ferrite filled apertures, Proc. IRE 44, 1463-1468.

Franz, W. (1954), Uber die greensche Funktionen des Zylinders under der Kugel: Zeitschrift fur Naturforschung 9a, 705-716.

Harley, T. B., and G. Tyras (1961), Transmission of electromagnetic waves through an ionized layer in the presence of a strong magnetic field, Proc. IRE 49, No. 12, 1822-1824.

Hodora, H. (1961), The use of magnetic fields in elimination of the en-entry radio blackout, Proc. IRE 49, 1825-1830.

Hodora, H. (Jan. 1963), Radiation from a gyroplasma sheathed aperture, IRE Trans. Ant. Prop. AP-1 1, No. 1, 2-12.

Shore, R., and G. Meltz (1962), Anisotropic plasma covered magnetic line source, IRE Trans. Ant. Prop. AP-10, 78-82.

Silver, S., and W. K. Saunders (Feb. 1950), The external field produced by a slot in an infinite circular cylinder, J. Appl. Phys. 21 , No. 5, 153-158.

Tyras, G., and G. Held (July 1958), Radiation from rectangular waveguide filled with ferrite, IRE Trans. Microwave Theory Tech. MTT-6, 268-277.

Wait, J. R. (1959), Electromagnetic radiation from cylindrical structures (Pergamon Press, Inc., New York, N.Y.).

Wait, J. R. (April-June 1961), Some boundary value problems involving plasma media, J. Res. NBS 65B, (Math. and Math. Phys.) No. 3, 137-150.

Wait, J. R. (Aug. 1963), Transverse propagation of electromagnetic waves in a cylindrically stratified plasma, Pro. Radio Physics Symp. University of California, ed. J. Brandstatter (Holden-Day, Inc., San Francisco, Calif.).

Yeh, C. (July 1964), Electromagnetic radiation from an arbitrary slot on a conducting cylinder coated with a uniform cold plasma sheath with an axial static magnetic field, Can. J. Phys. 42, 1369-1381.

(Paper 69D4-489) 